\title{
Manifestaciones clínicas y complicaciones de la fase aguda de bartonelosis o Fiebre de la Oroya en pacientes atendidos en el Hospital Nacional Cayetano Heredia.
}

\author{
Clinical manifestations and complications of the acute phase of bartonellosis or Oroya's Fever in \\ patients of the Hospital Nacional Cayetano Heredia. \\ Maguiña Vargas Ciro Peregrino ${ }^{1,2}$, Peña Collantes Renzo Benigno ${ }^{3}$, Ponce Alvarez Mario Carlos ${ }^{2,3}$, Quispe- \\ Chullo Teófilo Fernando ${ }^{3}$, Ventosilla López Palmira Ryquett².
}

\begin{abstract}
RESUMEN
Objetivos: Determinar las características clínicas, epidemiológicas y de laboratorio de la bartonelosis aguda, así como las complicaciones en una serie de pacientes atendidos en el Hospital Nacional Cayetano Heredia. Material y métodos: Estudio descriptivo de tipo serie de casos de pacientes mayores de 15 años con bartonelosis aguda hospitalizados en el Hospital Nacional Cayetano Heredia entre 1993 y 2006. Resultados: La edad promedio fue 27,8 años, 72,7\% fueron varones, 51,5\% procedía de Lima y 33,3\% de Ancash. Los síntomas principales fueron: fiebre $90,9 \%$, cefalea $87,9 \%$, hiporexia $75,5 \%$, baja de peso $63,6 \%$, e ictericia $57,6 \%$. Dos pacientes presentaron alteraciones en el fondo de ojo: retinitis exudativa y retinopatía hemorrágica. El hematocrito promedio fue 20,8\% y 63,6\% cursaron hematocrito $\leq 20 \%$. Hubo desviación izquierda en $24,2 \%$, hiperbilirrubinemia a predominio indirecto $62,5 \%$ e hipoalbuminemia (<3mg/dl) 56,5\%. Se presentaron complicaciones en 84,8\%, el 27,2\% requirió internamiento en UCI. Las complicaciones no infecciosas ocurrieron en $84,8 \%$, hematológicas $81,8 \%$ y gastrointestinales $78,7 \%$. Las complicaciones infecciosas se presentaron en 12,1\%. Los antibióticos más utilizados fueron ciprofloxacina $66,6 \%$ y cloranfenicol $42,4 \%$, se administró corticoides en 9,1\% y se transfundió paquete globular en $42,4 \%$. Conclusiones: Los pacientes de UCI tuvieron más complicaciones cardiovasculares, trombocitopenia y mayor severidad de la anemia. No se registró mortalidad. (Rev Med Hered 2008;19:87-95)
\end{abstract}

PALABRAS CLAVE: Bartonelosis, complicaciones, fiebre de la Oroya.

\footnotetext{
${ }^{1}$ Medico Infectólogo Tropicalista, Dermatólogo. Profesor Principal, Departamento de Medicina de la Universidad Peruana Cayetano Heredia. Médico Asistente del Departamento de Enfermedades Transmisibles y Dermatológicas del Hospital Nacional Cayetano Heredia. Lima, Perú.

${ }^{2}$ Instituto de Medicina Tropical Alexander von Humboldt. Universidad Peruana Cayetano Heredia. Lima, Perú.

${ }^{3}$ Médico Cirujano. Universidad Peruana Cayetano Heredia. Lima, Perú.
} 


\section{SUMMARY}

Objectives: To determine the clinical, epidemiological and laboratory of acute bartonellosis, as well as complications in a series of patients treated at the Hospital Nacional Cayetano Heredia. Material and methods: Descriptive study type series of patients aged over 15 years with acute bartonellosis hospitalized at the Hospital Nacional Cayetano Heredia between 1993 and 2006. Results: The mean age was 27.8 years, 72.7\% were male, $51.5 \%$ were from Lima and 33.3\% from Ancash. The main symptoms were: fever 90.9\%, headache $87.9 \%$, hyporexia $75.5 \%$, weight loss $63.6 \%$ and jaundice $57.6 \%$. Two patients showed abnormalities in the ophtalmoscopie: exudative retinitis and hemorrhagic retinopathy. The mean hematocrit was $20.8 \%$ and $63.6 \%$ had hematocrit $\leq 20 \%$. There was left shift in $24.2 \%$, indirect hyperbilirubinemia $62.5 \%$ and hypoalbuminaemia $56.5 \%$. Complications were present in $84.8 \%$ and $27.2 \%$ required placement in the ICU. Noninfectious complications occurred in $84.8 \%$, hematological $81.8 \%$ and gastrointestinal $78.7 \%$. Infectious complications were presented in $12.1 \%$. The most commonly antibiotics used were ciprofloxacin in $66.6 \%$ and chloramphenicol $42.4 \%$, corticoids were administered in 9,1\% and 42,4\% required red blood cell transfusion. Conclusions: ICU patients had more cardiovascular complications, thrombocytopenia and increased severity of anemia. There was no mortality. (Rev Med Hered 2008;19:87-95)

KEY WORDS: Bartonellosis, complications, Oroya’s fever.

\section{INTRODUCCIÓN}

La bartonelosis es una enfermedad de endemia ancestral, se presenta en el Perú, Ecuador y Colombia $(1,2)$. Presenta dos fases tipicas: anémica y eruptiva $(1,3)$. La fase anémica conocida como Fiebre de la Oroya es la forma de presentación más grave que puede llevar a la muerte del paciente sin un diagnóstico rápido y tratamiento oportuno (1).

Se ha reportado la existencia de un periodo de depresión del sistema inmune, que se cree no sólo se deba a una linfopenia sino también a déficit funcional $(4,5)$.

Las complicaciones de la bartonelosis aguda son frecuentes y pueden clasificarse en infecciosas y no infecciosas, haciendo que la letalidad en estos pacientes sea muy alta. Se han descrito complicaciones neurológicas, cardiovasculares, hematológicas y gastrointestinales (5-8). Una complicación hematológica poco descrita y recientemente reportada es la púrpura trombocitopénica que puede cursar con evolución favorable o seguir un curso tórpido (9).

La bartonelosis en la última década está extendiéndose a nuevas áreas de transmisión y el número de casos se ha incrementando notoriamente por lo que se considera como una enfermedad de importancia creciente en el Perú $(4,10,11)$.

Muchos aspectos de esta enfermedad aún no se han comprendido en su totalidad. El estudio tuvo como objetivo determinar las características clínicas, epidemiológicas y de laboratorio de la bartonelosis aguda, así como las complicaciones en una serie de pacientes atendidos en el Hospital Nacional Cayetano Heredia $(\mathrm{HNCH})$.

\section{MATERIAL Y MÉTODOS}

Estudio descriptivo de tipo prospectivo, serie de casos basados en las historias clínicas de pacientes evaluados desde 1980. La población estudiada incluyó a todos los pacientes mayores de 15 años con diagnóstico de bartonelosis en fase aguda atendidos en el servicio de Medicina y en el Departamento de Enfermedades Infecciosas, Tropicales y Dermatológicas (DEITD) del HNCH entre 1993 y 2006.

Los criterios de inclusión fueron:

- Edad mayor de 15 años.

- Diagnóstico de bartonelosis con al menos una manifestación clínica asociada con la fase aguda y con examen de frotis de sangre periférica o hemocultivo positivo para Bartonella bacilliformis o Western Blot o Reacción en Cadena de la Polimerasa (PCR) positiva para B. bacilliformis.

Se revisaron los libros de hospitalización y posteriormente las historias clínicas en los archivos de la oficina de estadística. Se consignaron los datos en una ficha clínica elaborada de acuerdo a los objetivos planteados y luego almacenados en una base de datos y procesadas utilizando el Programa estadístico SPSS v. 12.0 para Windows en español. Se determinaron 
frecuencias y porcentajes de las variables, y promedios y rangos para las variables continuas. Para la determinación de asociaciones se utilizó la Prueba Exacta de Fisher y la t de Student dependiendo del tipo de variable. Se consideró significativo un $\mathrm{p}<0,05$.

\section{RESULTADOS}

Se encontraron 56 pacientes, se excluyeron 19 por no cumplir los criterios de inclusión y 4 por no encontrar las historias clínicas en el archivo. Se incluyeron 33 pacientes, 11 habían sido referidos con diagnóstico establecido por frotis de lámina periférica. Los 33 pacientes tenían lámina periférica positiva para B. bacilliformis, dos hemocultivo positivo, dos western blot, y uno PCR.

Venticuatro fueron varones (72,7\%) y 9 mujeres (27,3\%). La edad promedio fue de 27,8 años (rango 16-76).

El 51,5\% (17 pacientes) procedía de Lima, 33,3\% de Ancash (Gráfico N¹). Quince pacientes (88,24\%) procedentes de Lima habían viajado a zonas endémicas en los últimos dos meses, de ellos el 73,3\% a Ancash.

Los síntomas principales al ingreso fueron: fiebre $90,9 \%$, cefalea $87,9 \%$, hiporexia $75,5 \%$, baja de peso $63,6 \%$, ictericia $57,6 \%$, sudoración $54,5 \%$, coluria
$54,5 \%$, náuseas 51,5\%, escalofríos 48,5\%, trastorno de conciencia $27,3 \%$ y palidez $27,3 \%$.

Diez pacientes (30\%) habían recibido terapia antibiótica previa siendo ampicilina, cloranfenicol y ciprofloxacina los antibióticos más usados. Un paciente tenía el antecedente de haber sido sometido a esplenectomía por púrpura trombocitopénica idiopática (PTI) 20 años antes y el 6\% tenían historia de tuberculosis pulmonar.

Los hallazgos del examen físico se muestran en la tabla $\mathrm{N}^{\circ} 1$. Los signos mas frecuentes fueron palidez 93,9\%, ictericia 69,7\%; linfoadenomegalias 48,5\%, hepatomegalia 51,5\% y esplenomegalia 27,3\%. Se realizó examen del fondo de ojo a 14 pacientes; el 85,7\% fueron normales. Un paciente tenía retinitis exudativa y otro retinopatía hemorrágica.

Los resultados de los exámenes de laboratorio se pueden observar en la tabla $\mathrm{N}^{\circ} 2$. El 63,6\% tuvo hematocrito $\leq 20 \%$, 24,2\% desviación izquierda, 85,7\% hiperbilirrubinemia (bilirrubina total $>1 \mathrm{mg} / \mathrm{dl}$ ), 62,5\% a predominio indirecto, 56,5\% albúmina $<3$ gr/dl y $2 /$ 4 pacientes Coombs directo positivo.

En trece pacientes no se cuantificó el índice parasitario, ni la forma del microorganismo. En los 20 pacientes restantes, se encontraron bacilos en 9 (45\%);

\section{Gráfico №1: Zonas de Procedencia}

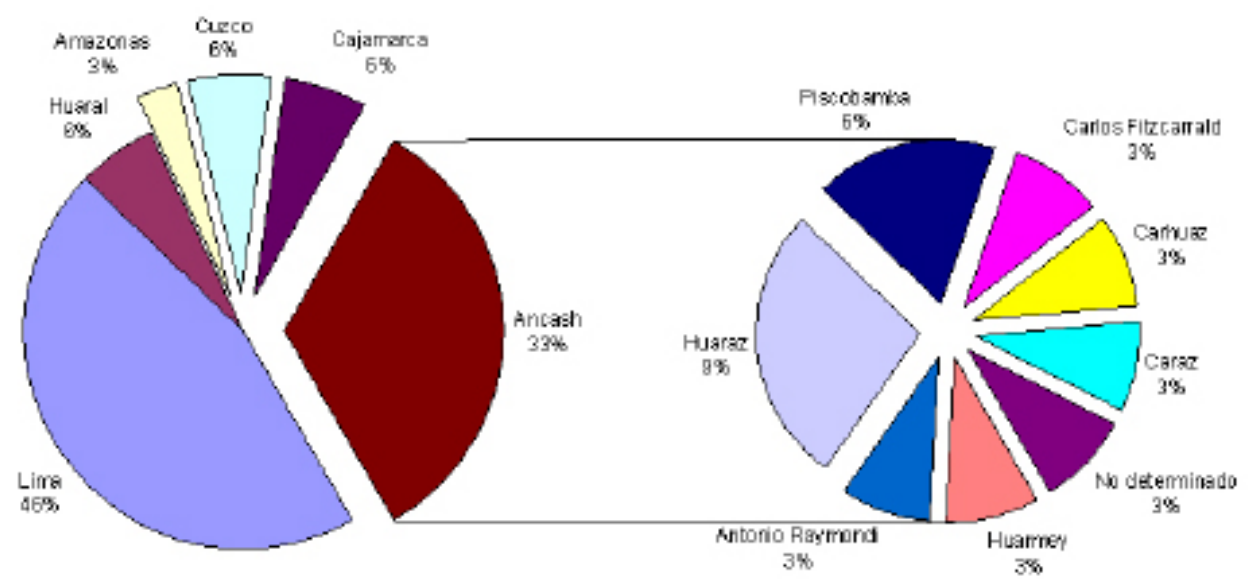

\begin{tabular}{|c|c|c|c|c|}
\hline 口Lima & 口 Huaral & 口Amazonas & 口Cuzos & acajamarca \\
\hline 口Huarmey & cAntonio Raymond & 口Huaraz & - Piscobamba & $\square$ Carlos Fitzcarad \\
\hline 口Carhuaz & DCaraz & a No deteminado & & \\
\hline
\end{tabular}


Tabla N³: EKG y estudios de imágenes.

Tabla Nº1: Hallazgos del examen físico $(n=33)$.

\begin{tabular}{|c|c|c|}
\hline & & n (\%) \\
\hline \multirow{3}{*}{ Piel } & Palidez & $31(93,9 \%)$ \\
\hline & Petequias & $2(6,1 \%)$ \\
\hline & Ictericia & $23(69,7 \%)$ \\
\hline \multirow{6}{*}{ Linfoadenomegalias } & Cervical & $9(27,3 \%)$ \\
\hline & Supraclavicular & $2(6,1 \%)$ \\
\hline & Inguinal & $10(30,3 \%)$ \\
\hline & Submaxilar & $2(6,1 \%)$ \\
\hline & Axilar & $4(12,1 \%)$ \\
\hline & Retroauricular & $2(6,1 \%)$ \\
\hline \multirow{5}{*}{ Sistema Respiratorio } & Alteración murmullo vesicular & $4(12,1 \%)$ \\
\hline & Crepitantes & $1(3,0 \%)$ \\
\hline & Subcrepitantes & $4(12,1 \%)$ \\
\hline & Sibilantes & $2(6,1 \%)$ \\
\hline & Tirajes & $1(3,0 \%)$ \\
\hline \multirow{6}{*}{ Sistema Cardiovascular } & Frote pericárdico & $1(3,0 \%)$ \\
\hline & Soplo cardiaco & $16(48,5 \%)$ \\
\hline & Reflujo hepatoyugular & $1(3,0 \%)$ \\
\hline & Ingurgitación yugular & $1(3,0 \%)$ \\
\hline & Edemas & $1(3,0 \%)$ \\
\hline & Bradicardia & $1(3,0 \%)$ \\
\hline \multirow{4}{*}{ Sistema Gastrointestinal } & Hepatomegalia & $17(51,5 \%)$ \\
\hline & Esplenomegalia & $9(27,3 \%)$ \\
\hline & Traube ocupado & $7(21,2 \%)$ \\
\hline & Dolor abdominal a la palpación & $4(12,1 \%)$ \\
\hline \multirow{2}{*}{ Sistema Nervioso } & Somnolencia & $4(12,1 \%)$ \\
\hline & Coma & $1(3,0 \%)$ \\
\hline
\end{tabular}

Tabla N²: Exámenes de laboratorio.

\begin{tabular}{|c|c|c|}
\hline & n (\%) & Media (Min - Máx) \\
\hline Hematocrito promedio (\%) & & $20,8(9-40)$ \\
\hline Hematocrito <36\% & 31/33 (93,9\%) & \\
\hline Hematocrito $=20 \%$ & 21/33 (63,6\%) & \\
\hline Recuento leucocitario $\left(\mathrm{mm}^{3}\right)$ & & $11145(2600-53200)$ \\
\hline Recuento leucocitario $=11000 / \mathrm{mm}^{3}$ & $11 / 33(33,3 \%)$ & \\
\hline Desviación izquierda & 8/33 (24,2\%) & \\
\hline Recuento reticulocitos (\%) & & $10,5(1-38)$ \\
\hline Índice de producción medular & & $3,6(0,36-9,2)$ \\
\hline Recuento de plaquetas $\left(10^{3} / \mathrm{mm}^{3}\right)$ & & $235(42-450)$ \\
\hline Recuento de plaquetas $<150000 / \mathrm{mm}^{3}$ & $5 / 25(20,0 \%)$ & \\
\hline Coombs directo positivo & $2 / 4(50,0 \%)$ & \\
\hline $\begin{array}{l}\text { Hemocultivo positivo para Bartonella } \\
\text { bacilliformis }\end{array}$ & $2 / 9(22,2 \%)$ & \\
\hline Hemocultivo positivo para gérmenes comunes & 2/16 (12,5\%) & \\
\hline Fosfatasa alcalina inicial (mg/dL) & & $272(41-732)$ \\
\hline Fosfatasa alcalina control (mg/dL) & & $274(74-583)$ \\
\hline Alaninoaminotransferasa (TGP)(UI/L) & & $60,6(15-216)$ \\
\hline Aspartatoaminotransferasa (TGO)(UI/L) & & $78,4(14-226)$ \\
\hline Bilirrubina total (mg/dL) & & $6,2(0,4-35)$ \\
\hline $\begin{array}{l}\text { Indirecta (mg/dL) } \\
\text { Directa (mg/dL) }\end{array}$ & & $\begin{array}{l}3,3(0,2-15,1) \\
2,8(0,2-22,6)\end{array}$ \\
\hline Bilirrubina = $1 \mathrm{mg} / \mathrm{dL}$ & 24/28 (85,7\%) & \\
\hline Hiperbilirrubinemia indirecta & $15 / 24(62,5 \%)$ & \\
\hline Proteínas totales (mg/dL) & & $6,1(4,6-7,8)$ \\
\hline Albúmina (mg/dL) & & $2,98(2,1-4,3)$ \\
\hline Proteínas totales $<6 \mathrm{mg} / \mathrm{dL}$ & $11 / 23(47,8 \%)$ & \\
\hline Albúmina <3 mg/dL & 13/23 (56,5\%) & \\
\hline Urea $(\mathrm{mg} / \mathrm{dL})$ & & $36,3(14,4-82)$ \\
\hline Urea $=40 \mathrm{mg} / \mathrm{dL}$ & 4/23 (17,4\%) & \\
\hline Creatinina (mg/dL) & & $0,9(0,39-1,58)$ \\
\hline Creatinina $>1 \mathrm{mg} / \mathrm{dL}$ & $6 / 30(20,0 \%)$ & \\
\hline
\end{tabular}

\begin{tabular}{|c|c|c|}
\hline & & n (\%) \\
\hline \multirow{5}{*}{$\begin{array}{l}\text { Electrocardiograma } \\
(\mathrm{n}=29)\end{array}$} & Taquicardia sinusal & $13(44,8 \%)$ \\
\hline & Normal & $11(37,9 \%)$ \\
\hline & Rotación antihoraria del eje & $2(6,9 \%)$ \\
\hline & Trastorno difuso de la despolarización & $2(6,9 \%)$ \\
\hline & Alternancia eléctrica & $1(3,4 \%)$ \\
\hline \multirow{8}{*}{$\begin{array}{l}\text { Radiografía de Tórax } \\
(\mathrm{n}=29)\end{array}$} & Normal & $12(41,4 \%)$ \\
\hline & Refuerzo parahiliar & $8(27,6 \%)$ \\
\hline & Aumento de la trama broncovascular & $4(13,8 \%)$ \\
\hline & Cardiomegalia & $3(10,3 \%)$ \\
\hline & Derrame pleural y consolidación & $1(3,4 \%)$ \\
\hline & Infiltrado alveolar & $1(3,4 \%)$ \\
\hline & $\begin{array}{l}\text { Redistribución de flujo, ausencia de } \\
\text { transparencia apical derecha e hilio } \\
\text { engrosado de aspecto inflamatorio }\end{array}$ & $1(3,4 \%)$ \\
\hline & Infiltrado intersticial & $1(3,4 \%)$ \\
\hline \multirow{5}{*}{$\begin{array}{l}\text { Ecografía Abdominal } \\
(\mathrm{n}=22)\end{array}$} & Ascitis & $7(31,8 \%)$ \\
\hline & Hepatoesplenomegalia & $13(59,1 \%)$ \\
\hline & Esplenomegalia & $2(9,1 \%)$ \\
\hline & Hepatomegalia & $2(9,1 \%)$ \\
\hline & Normal & $2(9,1 \%)$ \\
\hline \multirow{11}{*}{$\begin{array}{l}\text { Ecocardiografía } \\
(\mathrm{n}=25)\end{array}$} & Normal & $13(52,0 \%)$ \\
\hline & Dilatación leve de aurícula izquierda & $4(16,0 \%)$ \\
\hline & Derrame pericárdico & $4(16,0 \%)$ \\
\hline & Derrame pericárdico leve & $3(12,0 \%)$ \\
\hline & Taponamiento cardiaco & $1(4,0 \%)$ \\
\hline & Insuficiencia tricuspídea leve & $2(8,0 \%)$ \\
\hline & Dilatación leve de ventrículo izquierdo & $2(8,0 \%)$ \\
\hline & $\begin{array}{l}\text { Fracción eyección disminuida y } \\
\text { engrosamiento septal }\end{array}$ & $1(4,0 \%)$ \\
\hline & Hipokinesia & $1(4,0 \%)$ \\
\hline & Hipertrofia ventricular derecha & $1(4,0 \%)$ \\
\hline & Regurgitación mitral leve & $1(4,0 \%)$ \\
\hline
\end{tabular}

formas cocoides en 6 (30\%) y cocobacilos en 5 (25\%). El índice parasitario inicial promedio fue 57,8\% (rango: 5-100); y el índice parasitario final promedio 9,5\% (rango: 0-67).

En nueve pacientes se realizó hemocultivo para $B$. bacilliformis, resultando dos positivos (22,2\%). Además, en 16 pacientes se realizó hemocultivo para gérmenes comunes, resultando dos positivos (12,5\%), aislandose Salmonella sp y Klebsiella sp.

La ecocardiografía fue anormal en el $48 \%$ de los pacientes a quienes se realizó el estudio; se encontró derrame pericárdico en 16\%, incluyendo una paciente con taponamiento cardiaco (Tabla $\mathrm{N}^{\circ} 3$ ).

En relación con el tratamiento, se utilizó monoterapia antibiótica en 14 pacientes: ciprofloxacina en 10/14 (71,4\%) y cloranfenicol en 4/14 (28,6\%) y asociación de antibióticos en 19 casos: cloranfenicol y penicilina G 3/19 (15,7\%); ciprofloxacina y betalactámicos 5/ 19 (26,3\%); ciprofloxacina y cloranfenicol 3/19 (15,7\%); ciprofloxacina y vancomicina $1 / 19$ (5,2\%); cloranfenicol, penicilina G y clindamicina 1/19 (5,2\%) entre otros. 
Tabla $N^{\circ} 4$ : Complicaciones $(n=33)$.

\begin{tabular}{llr}
\hline & & $\mathrm{n}(\%)$ \\
\hline \multirow{4}{*}{ Cardiovasculares } & Bradicardia sinusal & $1(3,0 \%)$ \\
& Cardiomegalia & $2(6,1 \%)$ \\
& Efusión pericárdica & $4(12,1 \%)$ \\
& Taponamiento cardiaco & $1(3,0 \%)$ \\
& Miocarditis & $5(15,2 \%)$ \\
& & $1(3,0 \%)$ \\
Gastrointestinales & Anasarca & $1(3,0 \%)$ \\
& Hepatomegalia & $1(3,0 \%)$ \\
& Ictericia & $21(63,6 \%)$ \\
& Esplenomegalia & $19(57,6 \%)$ \\
Neurológicas & Somnolencia & $14(42,4 \%)$ \\
& Coma & $5(15,2 \%)$ \\
& Retinopatía hemorrágica & $1(3,0 \%)$ \\
& Retinitis exudativa & $1(3,0 \%)$ \\
Aparato Respiratorio & $1(3,0 \%)$ \\
& Neumonía & $1(3,0 \%)$ \\
& Herrame pleural & $1(3,0 \%)$ \\
Hemoptisis & $1(3,0 \%)$ \\
Gineco-Obstétricas & Edema agudo de pulmón & $1(3,0 \%)$ \\
(n) 9) & Gestante & $21(63,6 \%)$ \\
& Ginecorragia & $2(22,2 \%)$ \\
& Probable anemia hemolítica & $1(11,1 \%)$ \\
& Anemia hemolítica autoinmune & $23(69,7 \%)$ \\
& Trombocitopenia & $2(6,1 \%)$ \\
& Anemia severa & $1(3,0 \%)$ \\
\hline & Azoemia &
\end{tabular}

En general, los antibióticos utilizados fueron: ciprofloxacina $66,6 \%$, cloranfenicol $42,4 \%$, clindamicina $18,1 \%$, ceftriaxona $12,1 \%$, penicilina G sódica $9,1 \%$, cotrimoxazol $6,1 \%$, cefazolina $3 \%$, ceftazidima 3\%, y vancomicina 3\%.

Se administró corticoides en 3 (9,1\%) pacientes: dos pacientes con anemia hemolítica autoinmune y otro en coma. El tiempo promedio fue 7 días (rango 2-10). Transfusión de paquete globular (PG) se realizó en el 42,4\%; recibiendo en promedio 2,8 PG por paciente (rango 2-5).

En el servicio de emergencia no hubo sospecha de bartonelosis aguda en el $24,2 \%$ de los casos; siendo catalogados como síndrome ictérico febril, síndrome anémico y proceso infeccioso generalizado.

Se presentaron complicaciones en $84,8 \%$ de los casos, ameritando internamiento en la Unidad de Cuidados Intensivos (UCI) o en la Unidad de ShockTrauma el 24,2\%. El tiempo de permanencia fue en promedio 3 días (rango de $1-5$ ).

Tabla Nº5: Comparación pacientes de UCI vs No UCI

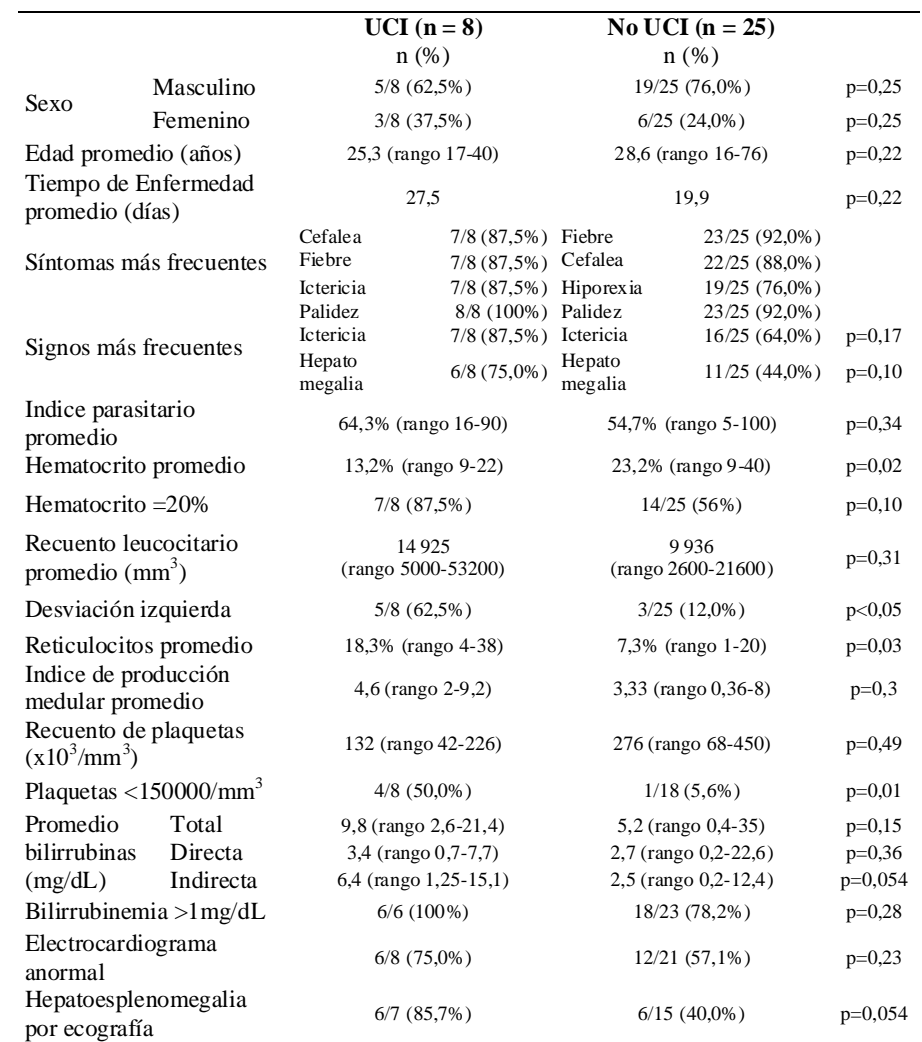


Tabla Nº6: Complicaciones en pacientes de UCI y No UCI

\begin{tabular}{|c|c|c|c|}
\hline Complicaciones & $\begin{array}{l}\text { UCI } \\
\mathbf{N}=8\end{array}$ & $\begin{array}{c}\text { No UCI } \\
\mathrm{N}=25\end{array}$ & \\
\hline Cardiovasculares & $6 / 8(75,0 \%)$ & $6 / 25(24,0 \%)$ & $p=0,01$ \\
\hline Efusión pericárdica & $2 / 8(25,0 \%)$ & $2 / 25(8,0 \%)$ & $\mathrm{p}=0,20$ \\
\hline $\begin{array}{l}\text { Taponamiento } \\
\text { Cardiaco }\end{array}$ & $1 / 8(12,5 \%)$ & $0 / 25(0 \%)$ & $\mathrm{p}=0,24$ \\
\hline $\begin{array}{l}\text { Insuficiencia Cardiaca } \\
\text { Congestiva }\end{array}$ & $2 / 8(25,0 \%)$ & $3 / 25(12,0 \%)$ & $\mathrm{p}=0,27$ \\
\hline Cardiomegalia & $1 / 8(12,5 \%)$ & $1 / 25(4,0 \%)$ & $\mathrm{p}=0,37$ \\
\hline Bradicardia sinusal & $1 / 8(12,5 \%)$ & $0 / 25(0 \%)$ & $\mathrm{p}=0,24$ \\
\hline Miocarditis & $1 / 8(12,5 \%)$ & $0 / 25(0 \%)$ & $\mathrm{p}=0,24$ \\
\hline Gastrointestinales & 8/8 (100\%) & $18 / 25(72,0 \%)$ & $\mathrm{p}=0,11$ \\
\hline Hepatomegalia & $7 / 8(87,5 \%)$ & $15 / 25(60,0 \%)$ & $\mathrm{p}=0,13$ \\
\hline Esplenomegalia & $4 / 8(50,0 \%)$ & $10 / 25(40,0 \%)$ & $\mathrm{p}=0,27$ \\
\hline Ictericia & $5 / 8(62,5 \%)$ & $14 / 25(56,0 \%)$ & $\mathrm{p}=0,30$ \\
\hline Neurológicas & $4 / 8(50,0 \%)$ & $4 / 25(16,0 \%)$ & $\mathrm{p}=0,06$ \\
\hline Somnolencia & $3 / 8(37,5 \%)$ & $2 / 25(8,0 \%)$ & $\mathrm{p}=0,07$ \\
\hline Coma & $1 / 8(12,5 \%)$ & $0 / 250 \%)$ & $\mathrm{p}=0,24$ \\
\hline Retinitis exudativa & $0 / 8(0 \%)$ & $1 / 25(4,0 \%)$ & $\mathrm{p}=0,75$ \\
\hline $\begin{array}{l}\text { Retinopatía } \\
\text { hemorrágica }\end{array}$ & $0 / 8(0 \%)$ & $1 / 25(4,0 \%)$ & $\mathrm{p}=0,75$ \\
\hline Respiratorias & $2 / 8(25,0 \%)$ & $0 / 25(0 \%)$ & $\mathrm{p}=0,053$ \\
\hline Hemoptisis & $1 / 8(12,5 \%)$ & $0 / 25(0 \%)$ & $\mathrm{p}=0,24$ \\
\hline Neumonía & $1 / 8(12,5 \%)$ & $0 / 25(0 \%)$ & $\mathrm{p}=0,24$ \\
\hline Derrame pleural & $1 / 8(12,5 \%)$ & $0 / 25(0 \%)$ & $\mathrm{p}=0,24$ \\
\hline $\begin{array}{l}\text { Edema agudo de } \\
\text { pulmón }\end{array}$ & $1 / 8(12,5 \%)$ & $0 / 25(0 \%)$ & $\mathrm{p}=0,24$ \\
\hline Gineco-obstétricas & $2 / 3(66,7 \%)$ & 1/6 (16,7\%) & $\mathrm{p}=0,21$ \\
\hline Gestantes & $1 / 3(33,3 \%)$ & 1/6 (16,7\%) & $\mathrm{p}=0,50$ \\
\hline Ginecorragia & $1 / 3(33,3 \%)$ & $0 / 6(0 \%)$ & $\mathrm{p}=0,33$ \\
\hline Hematológicas & $8 / 8(100 \%)$ & $20 / 25(80,0 \%)$ & $\mathrm{p}=0,22$ \\
\hline $\begin{array}{l}\text { Probable anemia } \\
\text { hemolítica }\end{array}$ & $7 / 8(87,5 \%)$ & $16 / 25(64,0 \%)$ & $\mathrm{p}=0,17$ \\
\hline $\begin{array}{l}\text { Anemia hemolítica } \\
\text { autoinmune }\end{array}$ & $1 / 8(12,5 \%)$ & $1 / 25(4,0 \%)$ & $\mathrm{p}=0,37$ \\
\hline Anemia severa & $7 / 8(87,5 \%)$ & $14 / 25(56,0 \%)$ & $\mathrm{p}=0,10$ \\
\hline Trombocitopenia & $4 / 8(50,0 \%)$ & $1 / 25(4,0 \%)$ & $\mathbf{p}<\mathbf{0 , 0 5}$ \\
\hline \multicolumn{4}{|l|}{ Otras complicaciones } \\
\hline Azoemia & $2 / 8(25,0 \%)$ & $0 / 25(0 \%)$ & $\mathrm{p}=0,053$ \\
\hline $\begin{array}{l}\text { Coinfección por } \\
\text { Salmonella sp. }\end{array}$ & $0 / 8(0 \%)$ & $2 / 25(8,0 \%)$ & $\mathrm{p}=0,28$ \\
\hline Toxoplasmosis & $0 / 8(0 \%)$ & $1 / 25(4,0 \%)$ & $\mathrm{p}=0,75$ \\
\hline Ascariosis & $1 / 8(12,5 \%)$ & $0 / 25(0 \%)$ & $\mathrm{p}=0,24$ \\
\hline
\end{tabular}

Las complicaciones se pueden observar el la tabla $\mathrm{N}^{\circ} 4$. Las más frecuentes fueron hematológicas $84,8 \%$, gastrointestinales $78,7 \%$, cardiovasculares $36,4 \%$ y gineco-obstétricas 33,3\%. Un paciente infestación por Ascaris lumbricoides, que cursó con síndrome de Loeffler, hemoptisis y neumonía.

Al comparar los pacientes admitidos en la UCI y aquellos que no; se encontró diferencia estadísticamente significativa en el hematocrito promedio, el recuento de reticulocitos promedio; la aparición de desviación izquierda y la frecuencia de plaquetopenia (Tabla $\mathrm{N}^{\circ} 5$ ). Además, se encontró diferencia significativa en la presencia de complicaciones cardiovasculares (Tabla $\mathrm{N}^{\circ} 6$ ).

\section{DISCUSIÓN}

Nosotros desde 1980 venimos estudiando prospectivamente la Enfermedad de Carrión y en esta nueva serie observamos predominancia de varones, hallazgo que coincide con algunos reportes tanto en población adulta como en población pediátrica $(1,6)$. Más de la mitad de pacientes procedían de Lima; sin embargo, hay que destacar que un gran porcentaje tenía el antecedente de haber viajado a zonas endémicas en los dos meses previos. 
La fiebre y la palidez fueron el síntoma y el signo más frecuentes, lo que concuerda con lo reportado por Pachas (4) y Maguiña (12). La hepatomegalia como signo al ingreso fue encontrada en $51,5 \%$, menor al encontrado por Maguiña (12); tal vez debido a que nuestra población está conformada solamente por adultos.

En el examen del fondo de ojo encontramos un caso de retinitis exudativa y otro de retinopatía hemorrágica. Se ha descrito retinopatía hemorrágica en estudios anteriores asociadas a anemia severa (13); sin embargo, retinitis exudativa no ha sido reportada previamente, por lo que se necesitaría contar con mayor cantidad de pacientes para poder determinar su frecuencia y características.

Hemos encontrado hipoalbuminemia en el 56,5\%, hallazgo similar al estudio de Arévalo (14)quien encontró $54 \%$ en UCI y al de Breña (6) quien encontró $68 \%$ en niños.

Sólo en dos pacientes se aisló Bartonella bacilliformis en el hemocultivo. De los que tuvieron hemocultivo negativo, un paciente había recibido ciprofloxacino antes del ingreso (15). El resto de hemocultivos negativos, puede deberse a un inadecuado uso de la técnica de toma de la muestra o a otros factores.

Cabe destacar la presentación de un caso inusual de bacteremia persistente en un paciente esplenectomizado, el índice parasitario de 90\% persistió post tratamiento inicial, esto podría explicarse por una inadecuada capacidad fagocítica para eliminar la $B$. bacilliformis sin ayuda del bazo. Luego de recurrencia del cuadro clínico en dos ocasiones, respondió al tratamiento prolongado con ciprofloxacina por más de 2 meses; este caso ha llevado a creer que el bazo es el principal órgano linfoide implicado en el reconocimiento inmunológico de B. bacilliformis (16), sin embargo el mecanismo por el cual se lleva a cabo aún no ha sido aclarado.

El antibiótico más usado fue ciprofloxacina tanto en monoterapia como en combinación, lo cual difiere de otras series en las que el fármaco más usado era el cloranfenicol tanto en adultos como en niños $(6,12)$. Desde hace varios años se describe a las fluoroquinolonas como mejor alternativa que el cloranfenicol por sus beneficios, es un antibiótico bactericida intracelular con buena respuesta in vitro sobre B. bacilliformis y otros gérmenes oportunistas $(10,17)$.

Se usaron corticoides en tres pacientes debido a las complicaciones que presentaron; en dos por anemia hemolítica Coombs positivo y otro por trastorno de conciencia y coma $(1,4)$. Un gran porcentaje de pacientes recibió transfusión de paquete globular, esto se asocia con la severidad de la anemia producida por la enfermedad, Breña también reporta un alto porcentaje de transfusión de paquete globular (6).

Es importante mencionar que no hubo sospecha diagnóstica de bartonelosis aguda al ingreso en $24,2 \%$, lo que coincide con lo descrito en otros estudios $(6,18)$; siendo confundido con otras enfermedades como malaria, hepatitis viral, leptospirosis y brucelosis, dentro de enfoques sindrómicos como ictérico febril, anémico y proceso infeccioso generalizado.

Los pacientes que ingresaron a UCI tuvieron mayor proporción de desviación izquierda, plaquetopenia y hematocrito más bajo, hallazgos similares a los de Arévalo(14); también presentaron mayor frecuencia de complicaciones cardiovasculares, dato no reportado previamente. Las complicaciones neurológicas y respiratorias fueron similares a lo descrito en otros estudios $(1,14)$.

Se encontraron complicaciones en un alto porcentaje de los pacientes, predominando las hematológicas como la anemia hemolítica, en dos fueron autoinmunes Coombs directo positivo. Este hallazgo es similar al encontrado por Hinojosa en el año 1977 en Huaraz (7), sin embargo, hay un estudio en el que no se encontró anemia hemolítica Coombs positivo (1).

La hepatomegalia y la ictericia fueron las complicaciones gastrointestinales más frecuentes. Breña encontró hepatomegalia en el 78\% (6). La frecuencia de ictericia es similar al de estudios previos $(6,12)$, sin embargo, Hinojosa solo encontró 5\% (7). Además encontramos esplenomegalia en un porcentaje considerable coincidiendo con el reporte de un brote ocurrido en Cuzco en el año 1998 (8).

Dentro de las complicaciones cardiovasculares la insuficiencia cardiaca congestiva y la pericarditis fueron las más frecuentes, pero menores al descrito por Franco quien las describe en $62 \%$ y $56 \%$, respectivamente (5). Es importante comentar que un caso presentó taponamiento cardiaco, que requirió de ventana pericárdica; el resultado de la biopsia no fue concluyente. Las complicaciones respiratorias se 
presentaron en un bajo porcentaje con respecto a reportes anteriores (19).

Las complicaciones neurológicas se presentaron en menor frecuencia al descrito en series anteriores, hay estudios que encuentran complicaciones neurológicas hasta en $68 \%(12,13)$.

Las complicaciones infecciosas ocurrieron en menor frecuencia que la descrita en otros estudios que encuentran hasta $36 \%$, siendo las infecciones bacterianas, las más frecuentes $(1,12)$. En nuestra serie solo tres pacientes tuvieron complicaciones infecciosas: dos por salmonelosis y uno por toxoplasmosis diseminada. Hubo un caso de infestación por $A$. lumbricoides con síndrome de Loeffler; la coinfestación parasitaria ha sido reportada previamente y usualmente se asocia a inmunosupresión (12). Esta es la primera vez que se reporta la coinfestación por Ascaris lumbricoides en la fase aguda de la bartonelosis.

Con respecto a bartonelosis y gestación, el estudio reporta dos casos asociados con derrame pericárdico, uno de ellos requirió drenaje quirúrgico por ventana pericárdica, ambos evolucionaron con respuesta favorable a los antibióticos y viabilidad del feto al final del tratamiento. Montoya reporta $25 \%$ de complicaciones en esta población (8), mientras que López señala 48\% de complicaciones en gestantes en un estudio reciente y ocurrencia de óbito fetal en $4 \%$ (20).

Un hecho que llama la atención es que la mortalidad fue $0 \%$, a diferencia de otros estudios que encuentran 6 y 9\% de mortalidad $(6,12)$. La disminución de la mortalidad podría estar en relación con los cambios en el manejo antibiótico y de las complicaciones en un Centro de referencia para bartonelosis como el DEITD del HNCH y el Instituto de Medicina Tropical Alexander Von-Humboldt de la UPCH. Sin embargo, es necesario mencionar que un paciente fallecido el 2005, no fue incluido en la base de datos debido a que falleció en menos de 24 horas de permanencia en la Unidad de Trauma Shock de Emergencia del HNCH.

Podemos apreciar que por tratarse de pacientes hospitalizados hubo una alta frecuencia de complicaciones, mayor en los que ingresaron a la UCI, en su mayoría no infecciosas y con mortalidad cero, en relación probable con cambios en los esquemas terapéuticos y mejoras en el soporte vital.
Se requiere de nuevas investigaciones prospectivas a fin de dar respuesta a algunas interrogantes en las complicaciones, sobre todo las neurológicas y cardiovasculares; además de estudios comparativos del enfoque terapéutico de esta enfermedad.

\section{Correspondencia:}

Mario Carlos Ponce Alvarez

Las Rosas 260-El Remanso La Molina

Lima-Perú

Correo electrónico: mariocponce@gmail.com

\section{REFERENCIASBIBLIOGRÁFICAS}

1. Maguiña C, Gotuzzo E. Bartonellosis New and old. Infect Dis Clin North Am 2000; 14(1): 1-22.

2. Allison MJ, Pezzia A, Gerszten E, Mendoza D. A case of Carrion's disease associated with human sacrifice from the Huari culture of Southern Peru. Am J Phys Anthropol 1974; 41(2): 295-300.

3. Walker D, Guerra H, Maguiña C. Bartonellosis. En: Guerrant RL, Walker D, Weller P, editors. Tropical Infectious Diseases, Principles, Pathogens, and Practice. 1st ed. Philadelphia: Churchill Livingstone; 1999. p. 492-497.

4. Pachas P. Epidemiología de la bartonelosis en el Perú. Módulos Técnicos-Serie Documentos Monográficos. Lima, PE: Oficina General de Epidemiología/Instituto Nacional de Salud; 2000.

5. Franco V. Complicaciones cardiovasculares de la fase aguda de la bartonelosis en el Hospital Nacional Cayetano Heredia entre los años 1987-1997. Tesis para optar grado de Bachiller. Lima, Perú. Universidad Peruana Cayetano Heredia, 1998.

6. Breña J. Bartonellosis aguda en Niños. Estudio de una serie de casos en el Instituto Especializado de Salud del Niño y el Hospital Nacional Cayetano Heredia en el periodo 1993-2003.Trabajo de Investigación para optar el título de Médico-Cirujano. Lima, Perú. Universidad Peruana Cayetano Heredia, 2004.

7. Hinojosa W. Estudio clínico epidemiológico sobre 76 casos de bartonelosis en el Callejón de Huaylas. Informe memoria de SECIGRA 1977.

8. Montoya M, Maguiña C, Vigo B, et al. Brote epidémico de Enfermedad de Carrión en el Valle Sagrado de los Incas (Cuzco). Bol Soc Per Med Int 1998;11:170-176.

9. López D, Menacho J, Norabuena R, Romero O, Maguiña C. Púrpura Trombocitopénica y Bartonellosis aguda (enfermedad de Carrión) en Huaraz, Perú. Acta Med Per 2006; 23(1): 6-11.

10. Huarcaya E, Maguiña C, Torres R, Rupay J, Fuentes L. Bartonelosis (Carrion's Disease) in the pediatric population of Peru: An overview and updates. Braz J Infect Dis 2004; 8(5): 331-339. 
11. Ministerio de Salud. Vigilancia epidemiológica de la bartonelosis o Enfermedad de Carrión en el Perú. Dirección General de Epidemiología. Bol. Epidemiol 2006; 15(37).

12. Maguiña C, García P, Gotuzzo E, Cordero L, Spach D. Bartonellosis (Carrión's Disease) in the Modern Era. Clin Infect Dis 2001; 33: 772-779.

13. Maguiña C, Acosta R, Gotuzzo E, et al. Compromiso del sistema nervioso central en la enfermedad de Carrión. Revista Neuropsiquiatría 1996; 59: 3-25.

14. Arévalo J, Borasino J, Maguiña C, López D, Penacho J, Orihuela A. Bartonelosis o Fiebre de la Oroya en la Unidad de Cuidados intensivos del Hospital Víctor Ramos Guardia de Huaraz. XIV Congreso Nacional de Medicina Interna; 2006 Oct 26-29; Lima, Perú: Sociedad Peruana de Medicina Interna; 2006.

15. Novelli S. Determinación de la susceptibilidad antibiótica de 14 antibióticos para Bartonella bacilliformis por métodos microbiológicos e inmunohistoquímicos Tesis bachiller. Lima, Perú. Universidad Peruana Cayetano Heredia, 2004.
16. Henríquez C, Hinojosa JC, Ventosilla P, et al. Report of

unusual case of persistent bacteremia by Bartonella bacilliformis in a splenectomized patient. Am J Trop Med Hyg 2004; 71(1): 53-55.

17. Rolain JM, Brouqui P, Koehler JE, Maguiña C, Dolan MJ, Raoult D. Recommendations for treatment of human infections caused by Bartonella Species. Antimicrobian Agents and Chemotherapy 2004; 48(6): 1921-1933.

18. Maguiña C. Estudio de 23 casos de bartonellosis humana en San Marcos, Ancash. Diagnóstico 1981; 7 (6): 257-266.

19. Pachas P. Situación actual de la Enfermedad de Carrión en la DIRES Ancash hasta la semana epidemiológica No 23 de 1999. Boletín epidemiológico. Lima, Peru: Oficina General de Epidemiología/ Instituto Nacional de Salud; 1999.

20. López D, Giraldo M, Maguiña C. Complicaciones ginecoobstétricas en la bartonelosis aguda: 50 casos observados en Caraz, Ancash. Acta Med Per 2006; 23(3): 148-151. 